EUROPEAN JOURNAL OF PURE AND APPLIED MATHEMATICS

Vol. 11, No. 1, 2018, 284-298

ISSN 1307-5543 - www.ejpam.com

Published by New York Business Global

\title{
Performance of linear discriminant analysis using different robust methods
}

\author{
Mufda J. Alrawashdeh ${ }^{1, *}$, Taha Radwan ${ }^{1,2}$ and Khalid Abunawas ${ }^{1}$ \\ ${ }^{1}$ Department of Mathematics, College of Sciences and Arts, Al-Rass, Qassim University, \\ Kingdom of Saudi Arabia. \\ ${ }^{2}$ Department of Mathematics and Statistics, Port Said University, Port Said, Egypt
}

\begin{abstract}
This study aims to combine the new deterministic minimum covariance determinant (DetMCD) algorithm with linear discriminant analysis (LDA) and compare it with the fast minimum covariance determinant (FastMCD), fast consistent high breakdown (FCH), and robust FCH $(\mathrm{RFCH})$ algorithms. LDA classifies new observations into one of the unknown groups and it is widely used in multivariate statistical analysis. The LDA mean and covariance matrix parameters are highly influenced by outliers. The DetMCD algorithm is highly robust and resistant to outliers and it is constructed to overcome the outlier problem. Moreover, the DetMCD algorithm is used to estimate location and scatter matrices. The DetMCD, FastMCD, FCH, and RFCH algorithms are applied to estimate misclassification probability using robust LDA. All the algorithms are expected to improve the LDA model for classification purposes in banks, such as bankruptcy and failures, and to distinguish between Islamic and conventional banks. The performances of the estimators are investigated through simulation and actual data..
\end{abstract}

Key Words and Phrases: DetMCD, FastMCD, Financial Ratios, Linear Discriminant Analysis, Orthogonalized GnanadesikanKettenring, FCH, RFCH.

\section{Introduction}

Linear discriminant analysis (LDA) is widely used in multivariate statistical techniques for data analysis. Rules that describe separation among groups are obtained through LDA. Variables are assumed to be normally distributed with the equal covariance matrix $\sum$. LDA is highly sensitive to outlier observations. Hence, estimating LDA parameters using the classical approach will affect the values of parameters. Robust estimators have been proposed to limit the effects of outlier observations, and certain methods have been presented to overcome the outlier problem, such as the high breakdown criterion developed by Hawkins and McLachlan [11]. Croux et al. [5] investigated the classification efficiencies

${ }^{*}$ Corresponding author.

Email addresses: mufdajr@yahoo.com (M. J. Alrawashdeh), taha_ali_2003@hotmail.com (T. Radwan) and Xsx6666@gmail.com (Kh. Abunawas) 
of robust procedures with respect to the classical method. Rousseeuw [23] introduced the minimum covariance determinant (MCD) estimator. Rousseeuw and Driessen [25] developed a new estimator called fast minimum covariance determinant (FastMCD), which is a highly robust estimator for observing outliers, to fill the gap in contaminated datasets. Todorov [27] constructed the robust Wilks lambda, which is uninfluenced by contaminated data, based on FastMCD to avoid the outlier problem. FastMCD has been applied to LDA (He and Fung [12]; Hubert and van Driessen [14]) and to different aspects of science (Hubert et al. [16]). FastMCD is also used in many multivariate techniques, such as the principle component analysis (Croux and Haesbroeck [7]; Hubert et al. [17]), factor analysis (Pison et al. [22]), classifications, and clustering techniques. In a multivariate time series, Croux et al. [6] proposed the robust exponential smoothing of a multivariate time series to enhance the robustness of estimates for contaminated data. Different techniques and approaches use features of MCD to improve the robustness of parameter estimation. Hubert and Rousseeuw [15] presented a robust regression method for continuous situations and binary regression. Croux and Dehon [4] used robust canonical correlation. Hubert and Branden [13] introduced robustified versions of the SIMPLS algorithm. A robust multivariate calibration model was used by Hubert and Verboven [19], and a robust error in variable regression was used by Fekri and Ruiz-Gazen [9]. The MCD algorithm was used for a genetic algorithm by Wiegand et al. [29]). Hubert et al. [18] used a new estimator deterministic algorithm for robust location and scatter, called deterministic minimum covariance determinant (DetMCD), and compared it with two estimators, namely, FastMCD and orthogonalized GnanadesikanKettenring (OGK), of Maronna and Zamar [20]. The new estimator uses the same iteration as FastMCD but does not draw random subsets, whereas FastMCD draws random subsets of size $p+1$ and is required to draw several times to obtain at least one subset that is free from outliers. DetMCD exhibited better performance and was faster than the other two estimators in estimating location and scatter matrices. Olive and Hawkins [21] proposed an easy method for computing $\sqrt{n}$ consistent outlier resistant estimators that can be used for inference and adopted numerous applications, including outlier detection and diagnostics, to determine whether data distribution is elliptically contoured. Olive and Ye [30] used three robust estimators of multivariate location and dispersion and then applied one of these estimators to create a robust method for canonical correlation analysis. One of these methods is the fast consistent high breakdown (FCH) estimator, which is fast, consistent, and highly resistant to outliers. The current work aims to combine DetMCD with LDA and compare it with the FastMCD, FCH, and robust FCH (RFCH) algorithms through simulation and actual data. Three approaches, namely, pooled covariance (PCOV), POBS, and minimum within-group covariance determinant (MWCD), are applied to improve the initial covariance estimate $\sum_{0}$ for all the estimators used in this study. The performance of this LDA is evaluated based on these estimators. Our analysis indicates that DetMCD performs better than the other estimators for the raw and reweighted versions. 


\section{LDA}

Our proposed datasets of actual and generated data $p$ variables measured in $n$ observations may be summarized as the $n \times p$ matrix $X=\left(x_{i j}\right)$, where $x_{i j}$ denotes the expression level of $p$ variables in observations $i=1,2, \ldots, n_{j}$ that are sampled from $l$ different populations $\pi_{1}, \pi_{2}, \ldots, \pi_{l}$.

In the LDA setting, membership probability is estimated for each observation with respect to the population.

The data are sampled from $l$ populations, and each population has $n_{j}$ observations, $j=$ $1,2, \ldots, l . \sum_{j=1}^{l} n_{j}=n$ observations can be denoted by $\left\{x_{i j}=j=1,2, \ldots, l, i=1,2, \ldots, n\right\}$.

LDA has $\mu_{j}, \Sigma_{j}$, and $p_{j} . \mu_{j}$ is the mean, $\Sigma_{j}$ is the covariance matrix, and $p_{j}$ is the membership probability for each population $\pi_{j}$. LDA assumes a common covariance matrix $\Sigma$; all the parameters are unknown in practice and must be estimated from the sample data. In general, LDA parameters are estimated empirically, which leads to inaccurate values because LDA is highly influenced by outliers. All the parameters must be estimated based on robust estimators to overcome the outlier problem, thereby requiring high-performance robust estimators.

The robust LDA (RLDA) rule is expressed as follows:

$$
\begin{aligned}
& \text { Allocate } x \text { to } \pi_{j} \text { if } \widehat{d}_{k}^{R L}(x)>\widehat{d}_{j}^{R L}(x) \text { for } j=1,2, \ldots, g, j \neq k \text { with } \\
& \widehat{d}_{j}^{R L}(x)=\mu_{j}^{t} \Sigma^{-1} x-\frac{1}{2} \mu_{j}^{t} \Sigma^{-1} \mu_{j}+\ln \left(p_{j}\right),
\end{aligned}
$$

where $\Sigma$ is the common covariance matrix with mean $\mu_{j}$ and prior probability $p_{j}$. For the estimates of the membership probability $p_{j}$ in Eq. (1), we discuss two well-known choices. Either $p_{j}$ is considered constant over all populations, thereby yielding $p_{j}=1 / L$ for each $j$, or it is estimated as the relative frequencies of the observations in each group, thereby yielding $p_{j}=n_{j} / n$.

If $\tilde{n}_{j}$ denotes the number of non-outliers in group $j$ and $\tilde{n}=\sum_{j=1}^{l} \tilde{n}_{j}$, then the membership probability is robustly estimated as follows:

$$
\overbrace{P_{j}}^{R L}=\frac{\tilde{n}_{j}}{\tilde{n}}
$$

As previously mentioned, the LDA parameters are unknown and have to be estimated. All the estimators will be used to estimate the LDA parameters to apply LDA to the estimation of the misclassification probability. 


\section{DetMCD estimator}

The DetMCD algorithm starts by standardizing data to obtain the standardized $Z$. Each variable $X_{j}$ will be subtracted from the median and divided by the $Q_{n}$ scale estimator (Rousseeuw and Croux [24]). This standardization enables the equivariance of algorithm location and scale. The standardized dataset is denoted by the $n \times p$ matrix $Z$ with row $z_{i}^{t}(i=1,2, \ldots, n)$ and column $z_{j}(j=1,2, \ldots, p)$.

Six initial estimates of $\mu_{k}(z)$ and $\Sigma_{k}(z)$, where $(k=1,2, \ldots, 6)$, represent the mean and covariance matrix, respectively, of $Z$. Each $S_{k}$ estimator computes the covariance or correlations of matrix $Z$.

\section{Six initial scatter estimators}

(i) $S_{1}$ is computed by the hyperbolic tangent of each column of $Z, Y_{j}=\tanh \left(Z_{j}\right)$ for $j=1, \ldots, p$. This bounded function reduces the effect of large coordinatewise outliers. Then, the classical correlation matrix of $Y$ is computed to obtain $S_{1}=\operatorname{corr}(Y)$.

(ii) $S_{2}$ is computed by determining $R_{j}$, the rank of each column $Z_{j}$. Then, $S_{2}=\operatorname{corr}(R)$, which is the Spearman correlation matrix of $Z$.

(iii) $S_{3}$ is the normal score computed from $R_{j}$; that is, $T_{j}=\phi^{-1}\left(\left(R_{j}-1 / 3\right) /(n+1 / 3)\right)$, where $\phi(\cdot)$ is the normal cumulative distribution function. Then, $S_{3}=\operatorname{corr}(T)$.

(iv) $S_{4}$ is the scatter estimator computed based on the spatial sign covariance matrix (Visuri et al. [28]) and is defined as $k_{i}=z_{i} /\left\|z_{i}\right\|$ for all $i$. Then, $S_{4}=(1 / n) \sum_{i=1}^{n} k_{i} k_{i}^{T}$.

(v) $S_{5}$ is the first step of the BACON algorithm (Billor et al. [2]). The $\{n / 2\}$ standardized observation $z_{i}$ has the smallest norm and is used to compute the mean and covariance matrix.

(vi) A scatter estimate is the raw version of the OGK estimator. For $m(\cdot), s(\cdot)$, and the median, $Q_{n}$ is used for simplicity.

After standardizing the data and obtaining $c$, three steps are completed to obtain the covariance and mean of DetMCD.

(i) The matrix $E$ of the eigenvectors of $S_{k}$ is computed and $B=Z E$ is applied.

(ii) The center of $Z$ is estimated using $\Sigma_{k}(Z)=E L E^{T}$, where $L=\operatorname{diag}\left(Q_{n}^{2}(B 1), \ldots, Q_{n}^{2}\left(B_{p}\right)\right)$.

(iii) The covariance of $Z$ is estimated using sphere data, the coordinate-wise median is applied and transformed back, $\mu_{k}(Z)=\Sigma_{k}^{1 / 2}\left(\operatorname{med}\left(Z \Sigma_{k}^{1 / 2}\right)\right)$. 
For all the six estimates $S_{k},\left(\mu_{k}(Z), \Sigma_{k}(Z)\right)$ is used to compute the statistical distance, as follows:

$$
d_{i k}=d\left(\mu_{k}(Z), \Sigma_{k}(Z)\right)
$$

For the initial estimate $k, h_{\circ}=[n / 2]$ observations are taken with the smallest $d_{i k}$. Then, the statistical distances $d_{i k}^{*}$ for $h_{\circ}$ observations are computed. All $h$ observations $x_{i}$ are calculated with the smallest $d_{i k}^{*}$ for all the six estimates. The final step is the application of the concentration step (C-step) until convergence. The estimate with the smallest determinant is called the raw DetMCD. The final DetMCD is obtained by applying the reweighted FastMCD algorithm.

As previously mentioned, RLDA is constructed based on the DetMCD algorithm. RLDA is derived by inputting the location and scatter matrices obtained based on the DetMCD algorithm into LDA, as follows:

$$
\widehat{d}_{j}^{\operatorname{DetMCD}}(x)=\mu_{j}^{t} \Sigma^{-1} x-\frac{1}{2} \mu_{j}^{t} \Sigma^{-1} \mu_{j}+\ln \left(p_{j}\right) .
$$

Outliers in the data are flagged to robustify the location and scatter matrices. The robust distance for each observation $x_{i j}$ is computed from the group $\pi_{j}$ to estimate the membership probability, as follows:

$$
R D_{i j}^{D e t M C D}=\sqrt{\left(x_{i j}-\hat{\mu}_{j}\right)^{t} \Sigma^{-1}\left(x_{i j}-\hat{\mu}_{j}\right)} .
$$

Then, $x_{i j}$ is considered the outlier observation if and only if

$$
R D_{i j}>\sqrt{\chi_{p, 0.975}^{2}}
$$

Finally, the membership probability $\overbrace{P_{j}}^{\operatorname{Det} M C D}$ can be obtained using Formula (8) after applying the DetMCD estimator that is defined as follows:

$$
\overbrace{P_{j}}^{D e t M C D}=\frac{\tilde{n}_{j}}{\tilde{n}} .
$$

\section{FastMCD}

The main feature of the FastMCD algorithm is the C-step, where $\operatorname{det}\left(\Sigma_{\text {new }}\right) \operatorname{det}\left(\Sigma_{\text {old }}\right)$ with equality if $\operatorname{det}\left(\Sigma_{\text {new }}\right)=\operatorname{det}\left(\Sigma_{\text {old }}\right)$ (Rousseeuw and Driessen [25]). The application of the C-step will yield the sequence of determinants, which must converge in a finite number of steps. The final iteration cannot be guaranteed to be the minimum value of the MCD objective function. The FastMCD algorithm applied two C-steps to each initial subset, and only ten subsets with the smallest determinant for C-step are taken until initial convergence. Three approaches will be used with the FastMCD algorithm to estimate the 
mean and covariance matrix.

The same approach applied to the DetMCD algorithm to obtain RLDA, and $p_{j}^{R}$ is applied to the FastMCD algorithm, which is defined as follows:

$$
\widehat{d}_{j}^{\text {FastMCD }}(x)=\mu_{j}^{t} \Sigma^{-1} x-\frac{1}{2} \mu_{j}^{t} \Sigma^{-1} \mu_{j}+\ln \left(p_{j}\right) .
$$

The membership probability $p_{j}$ of the robust distance is defined as follows:

$$
R D_{i j}^{F a s t M C D}=\sqrt{\left(x_{i j}-\hat{\mu}_{j}\right)^{t} \Sigma^{-1}\left(x_{i j}-\hat{\mu}_{j}\right)} .
$$

If $x_{i j}$ is used to consider the outliers in Formula (6), then the membership probability of the FastMCD estimator is expressed as follows:

$$
\stackrel{\overbrace{P}^{F a s t M C D}}{=} \frac{\tilde{n}_{j}}{\tilde{n}}
$$

\section{6. $\mathrm{FCH}$}

The most practical estimators are used as a sequence of $n$ trial fits called initial estimator, $\left(\mu_{1}, \Sigma_{1}\right),\left(\mu_{2}, \Sigma_{2}\right), \ldots,\left(\mu_{n}, \Sigma_{n}\right)$. The initial estimator $\left(\mu_{i}, \Sigma_{i}\right)$ that minimizes the evaluation criterion will be used in the final estimator. The initial estimator obtained by the generated trial fits is called start. Then, the C-step technique will be applied.

We let $\left(\mu_{0, i}, \Sigma_{0, i}\right)$ be the $i$ th start and all $n$ Mahalanobis distances $D_{i}\left(\mu_{0, i}, \Sigma_{0, i}\right)$. The classical estimator $\left(\mu_{1, i}, \Sigma_{1, i}\right)$ is computed from $c_{n} \approx n / 2$ cases that correspond to the smallest distance. We continue the iteration for $k$ steps, thereby resulting in the following sequence: $\left(\mu_{0, j}, \Sigma_{0, j}\right)\left(\mu_{1, j}, \Sigma_{1, j}\right),\left(\mu_{2, j}, \Sigma_{2, j}\right), \ldots,\left(\mu_{k, j}, \Sigma_{n, k}\right)$. The values of $c_{n}$ and $k$ depend on the C-step estimator. The value of $k$ in the FastMCD estimator is 500, with randomly drawn elemental sets of $p+1$ cases as the start. The initial estimator with the smallest determinant is used for the final estimator. Hawkins and Olive [10] have a similar estimator.

The FCH estimator uses two estimators. The first estimator is the DGK estimator (Devlin, Gnanadesikan, and Kettenring [8]), which uses the classical estimator as the start. The second estimator is the median ball (MB) estimator, where the classical estimator is computed from cases with $D_{i}\left(M E D(X), I_{p}\right) \leq M E D\left(D_{i}\left(M E D(X), I_{p}\right)\right)$ as the start, and $M E D(X)$ is the coordinate-wise median. In case the DGK location estimator obtains a greater Euclidean distance from $M E D(X)$ than half of the data, then FCH will apply the median ball estimator. We let $\left(\mu_{0}, \Sigma_{0}\right)$ be the initial estimator used. Then, the estimator $(\mu, \Sigma)$ takes $\mu_{0}=\mu$ and $\Sigma=\frac{M E D\left(D_{i}^{2}\left(\mu_{0}, \Sigma_{0}\right)\right)}{\chi_{p, 0.5}^{2}} \Sigma_{0}$, where $\chi_{p, 0.5}^{2}$ is the 50 th percentile of the chi-square distribution with $p$ degrees of freedom.

The RLDA model is obtained using the FCH estimator in the raw and reweighted versions 
and is expressed as follows:

$$
{\widehat{d_{j}}}^{F C H}(x)=\mu_{j}^{t} \Sigma^{-1} x-\frac{1}{2} \mu_{j}^{t} \Sigma^{-1} \mu_{j}+\ln \left(p_{j}\right) .
$$

The membership probability $p_{j}^{R}$ of the robust distance is defined as follows:

$$
R D_{i j}^{F C H}=\sqrt{\left(x_{i j}-\hat{\mu}_{j}\right)^{t} \Sigma^{-1}\left(x_{i j}-\hat{\mu}_{j}\right)} .
$$

If $x_{i j}$ is used to consider the outliers in Formula (6), then the membership probability of the FCH estimator is expressed as follows:

$$
\stackrel{\overbrace{}}{P}_{j}^{F C H}=\frac{\tilde{n}_{j}}{\tilde{n}} .
$$

\section{Simulation study}

In this section, different algorithms are applied to estimate the LDA parameters using small and medium datasets. The simulation is similar to that of He and Fung [12].

All the estimators are used in the raw and reweighted versions to obtain the initial mean and covariance matrix, that is, $\mu_{0}$ and $\Sigma_{0}$, respectively. This estimator will yield a discriminate rule based on robust $d_{j}^{R L}\left(x, \mu_{0}, \Sigma_{0}\right)$. Then, the reweighted version will be obtained based on the robust distances (Rousseeuw and van Zomeren [26]), as follows:

$$
R D_{i j}=\sqrt{\left(x_{i j}-\hat{\mu}_{j, 0}\right)^{t} \Sigma_{0}^{-1}\left(x_{i j}-\hat{\mu}_{j, 0}\right)} .
$$

For each observation in group $j$,

$$
w_{i j}= \begin{cases}1 & \text { if } R D_{i j} \leq \sqrt{\chi_{p, 0.975}^{2}} \\ 0 & \text { otherwise }\end{cases}
$$

Three approaches presented by Hubert and van Driessen [14] are adopted to estimate the means and common covariance matrices for all the groups with raw and reweighted versions. The same approaches have been used to compare the robust and classical LDA (Alrawashdeh et al. [1]). These approaches are also applied to the estimators of the FastMCD, DetMCD, and FCH algorithms.

The first approach is direct and has been applied by Chork and Rousseeuw [3], where $\mu_{j}$ and $\Sigma_{j}$ are obtained by pooling the covariance matrix $\Sigma_{j, \text { Algorithm }}$ as follows:

$$
\widehat{\Sigma}_{P C O V}=\frac{\Sigma_{j=1}^{l} n_{j} \widehat{\Sigma}_{j_{\text {Algorithm }}}}{\sum_{j=1}^{l} n_{j}} .
$$


This approach will be denoted by PCOV for the raw version and PCOV-W for the reweighted version.

For the second approach, the concept is based on pooling the observations instead of the group covariance matrices. This approach was proposed by He and Fung [12], who used an S-estimator, and adopted by Hubert and van Driessen [14]. The number of groups in the simulation and that for other groups will follow the same pattern to simplify the notation of the three groups. In the three samples $A=\left(a_{11}, a_{21}, \ldots, a_{n_{1}, 1}\right), B=\left(b_{12}, b_{22}, \ldots, b_{n_{2}, 2}\right)$, and $C=\left(c_{13}, c_{23}, \ldots, c_{n_{3}, 3}\right), \mu_{A}, \mu_{B}$, and $\mu_{C}$ are the location estimators of the populations in the reweighted FastMCD. The pooled and shifted observations are expressed as follows:

$$
\begin{gathered}
Z=\left(z_{1}, z_{2}, \ldots, z_{n}\right)=\left(a_{11}-\mu_{A}, a_{21}-\mu_{A}, \ldots, a_{n_{1}, 1}-\mu_{A}, b_{12}-\mu_{B}, b_{22}-\mu_{B}, \ldots, b_{n_{2}, 2}-\mu_{B}\right. \\
\left., c_{13}-\mu_{C}, c_{23}-\mu_{C}, \ldots, c_{n_{3}, 3}-\mu_{C}\right) .
\end{gathered}
$$

The covariance matrix $\Sigma_{z}$ is estimated as the reweighted FastMCD of the scatter matrix of $z$. The location $\mu_{z}$ is estimated by the MCD estimator. $\mu_{z}$ is used to upgrade the locations of $\mu_{j}$ to obtain $\widehat{\mu}_{a}=\mu_{a}+\mu_{z}, \widehat{\mu}_{b}=\mu_{b}+\mu_{z}$, and $\widehat{\mu}_{c}=\mu_{c}+\mu_{z}$. The observations in this approach are pooled instead of the covariance matrices. Hence, RLDA is denoted by POBS for the raw version and POBS-W for the reweighted version.

The third method is a combination of the two previous methods. This method aims to derive a fast approximation of the MWCD criterion (Hawkins and McLachlan [11]). Instead of performing the same adjustment for each group, $h$ is identified from all observations with set size $n$, where the covariance matrix $\Sigma_{H}$ of $h$ has a minimal determinant. Then, the covariance matrix of $H$ ( $h$ out of $n$ ) is obtained. The approach for the three groups is as follows.

(i) The canters of the groups are estimated.

(ii) The observations are shifted and pooled to obtain $z$ 's that are the same as those in the second approach using the FastMCD estimator.

(iii) Let $H$ be $h$ out of $n$ based on the minimized FastMCD estimator.

(iv) The subset $H$ is partitioned into $H_{A}, H_{B}$, and $H_{C}$, which contain observations from $A, B$, and $C$, respectively.

(v) The mean of all the groups is estimated as $\mu_{A}, \mu_{B}$, and $\mu_{C}$.

In this approach, RLDA is denoted based on the MWCD for the raw version or MWCD$\mathrm{W}$ for the reweighted version. 


\section{Simulation result}

Through the simulation study, we compare the performances of the three approaches in estimating the initial values for the mean and covariance matrix and then apply the obtained values to FastMCD, DetMCD, and FHC to obtain the misclassification probabilities. We use the raw and reweighted versions for all the algorithms. The RLDA rule is applied using settings similar to those in the study of He and Fung [12]:

$$
\begin{aligned}
A \Rightarrow & \pi_{1}: 400 N_{3}(0, I) \\
& \pi_{2}: 400 N_{3}(1, I) \\
B \Rightarrow & \pi_{1}: 400 N_{3}(0, I)+50 N_{3}\left(5,\left(1 / 0.25^{2}\right) I\right) \\
& \pi_{2}: 400 N_{3}(1, I)+50 N_{3}\left(-4,\left(1 / 0.25^{2}\right) I\right) \\
C \Rightarrow & \pi_{1}: 80 N_{3}(0, I)+20 N_{3}\left(\left(1,\left(1 / 0.25^{2}\right) I\right)\right. \\
& \pi_{2}: 80 N_{3}(1, I)+20 N_{3}\left(\left(-1,\left(1 / 0.25^{2}\right) I\right)\right. \\
D \Rightarrow & \pi_{1}: 200 N_{3}(0, I)+25 N_{3}((1,(1 / 0.075) I) \\
& \pi_{2}: 200 N_{3}(1, I)+25 N_{3}((-1,(1 / 0.075) I) \\
E \Rightarrow & \pi_{1}: 300 N_{3}(0, I)+10 N_{3}\left(5,0.25^{2} I\right) \\
& \pi_{2}: 150 N_{3}(1, I)+5 N_{3}\left(-10,0.25^{2} I\right)
\end{aligned}
$$

where $I$ is the 3D identity matrix, the groups labeled as A, B, C, D and E, each group has to different cases $\pi_{1}$ and $\pi_{2}$. The membership probability is calculated based on Formula (8) for $l$ in consideration of the two algorithms. The classification rule (Eq. (1)) is robustified as the Fisher discriminant rule, which expressed as follows:

$$
x \in \pi_{1} i f\left(\widehat{\mu}_{1}-\widehat{\mu}_{2}\right)^{t} \Sigma^{-1}\left(x-\left(\widehat{\mu}_{1}-\widehat{\mu}_{2}\right) / 2\right)>0
$$

and $x \in \pi_{2}$ otherwise.

Figure 1 presents only the first 100 observations from the groups of Case C. The figure shows the scatter of data in Case $\mathrm{C}$, in which both groups have outliers. The effects of the outliers on data homogeneity and on the parameters of the discriminate rules that will be used to estimate values are clearly shown. Graphs C1 and C2 show a cut and abnormal spread for the observations.

This study aims to compare DetMCD with FastMCD and FCH using three approaches, that is, PCOV, POBS, and MWCD, for all algorithms at the raw and reweighted versions. As shown in Table 1 and Table 2, the DetMCD estimator clearly increases the efficiency of the discriminate rules in estimating the error rate of classification. Moreover, DetMCD performs better than the FastMCD and FCH estimators for the raw and reweighted versions. DetMCD for the raw and reweighted versions are more accurate in estimating the misclassification probabilities of data compared with the other two estimators. The second part of this comparative study will use the three approaches for the three estimators. The PCOV approach obtains the best estimated values for MP under the raw version, whereas the POBS approach achieves the best result for the reweighted version. DetMCD increases 

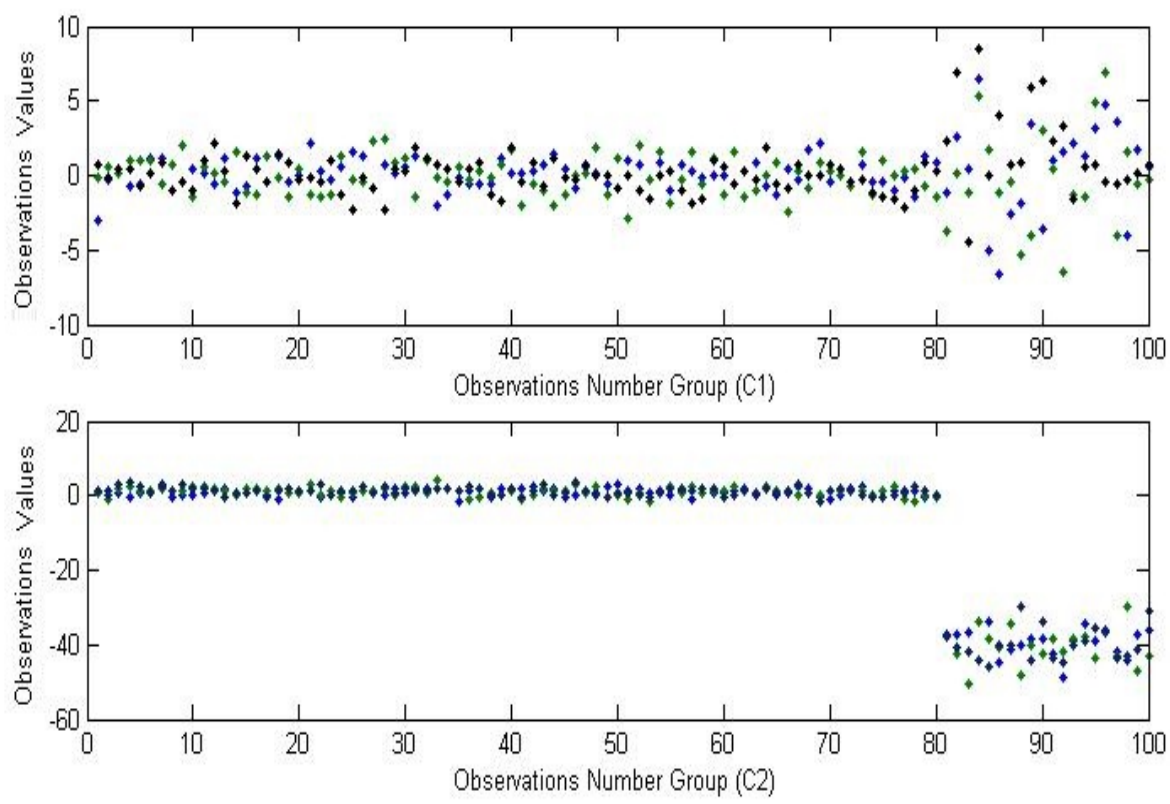

Figure 1: Observations Number Group of $\mathrm{C} 1$ and $\mathrm{C} 2$

the efficiency of the discriminate estimates compared with the other two estimators. In the two versions, the DetMCD algorithm obtains more accurate values for MP.

The DetMCD estimator values are more accurate and less than $10 \%$ for all the cases in the simulation. The data in Case A contain an uncontaminated dataset and the estimated values are comparable for both versions performance groups, except the MWCD for FastMCD and RFCH. The datasets for Cases B, C, D, and E are generated from contaminated datasets with different percentages of outliers for each case and group. Groups $\mathrm{A}$ and $\mathrm{B}$ are generated with the same number of observations. Case B is generated with $25 \%$ outlier observations. The outliers influence the estimator rules, where the estimated values of case B are 0.0751 and 0.0559 for the raw and reweighted versions, respectively,

Table 1: Mean $\mu$ for the misclassification probabilities of the RLDA rules for 500 replications based on the DetMCD, FastMCD, and FCH algorithms for the raw version

\begin{tabular}{|l|l|l|l|l|l|l|l|l|l|l|}
\hline Algorithm & \multicolumn{4}{l}{ FastMCD } & \multicolumn{4}{l|}{ DetMCD } & \multicolumn{4}{l|}{ FCH } \\
\hline Version & Raw & \multicolumn{4}{l|}{ Raw } & Raw & \\
\hline Approach & PCOV & POBS & MWCD & PCOV & POBS & MWCD & PCOV & POBS & MWCD \\
\hline Group A & 0.1967 & 0.1951 & 0.2662 & 0.0103 & 0.0211 & 0.0915 & 0.2151 & 0.1764 & 0.2235 \\
\hline Group B & 0.3145 & 0.3135 & 0.1314 & 0.0883 & 0.0751 & 0.1214 & 0.2945 & 0.3167 & 0.1736 \\
\hline Group C & 0.3651 & 0.3626 & 0.493 & 0.0998 & 0.102 & 0.1155 & 0.3476 & 0.3678 & 0.3987 \\
\hline Group D & 0.2785 & 0.2767 & 0.512 & 0.0963 & 0.0998 & 0.1089 & 0.3164 & 0.3023 & 0.4728 \\
\hline Group E & 0.2965 & 0.2944 & 0.3678 & 0.0247 & 0.0499 & 0.1169 & 0.2993 & 0.3284 & 0.3786 \\
\hline
\end{tabular}


Mufda J. Alrawashdeh, Taha Radwan and Khalid Abunawas / Eur. J. Pure Appl. Math, 11 (1) (2018), 284-298 294

Table 2: Mean $\mu$ for the misclassification probabilities of the RLDA rules for 500 replications based on the DetMCD, FastMCD, and FCH algorithms for the reweighted version

\begin{tabular}{|c|c|c|c|c|c|c|c|c|c|}
\hline Algorithm & \multicolumn{3}{|c|}{ FastMCD } & \multicolumn{3}{|c|}{ DetMCD } & \multicolumn{3}{|l|}{$\overline{\mathrm{RFCH}}$} \\
\hline \multirow{3}{*}{$\begin{array}{l}\text { Version } \\
\text { Approach }\end{array}$} & \multicolumn{3}{|c|}{ Reweighted } & \multicolumn{3}{|c|}{ Reweighted } & \multicolumn{3}{|c|}{ Reweighted } \\
\hline & PCOV- & POBS- & MWCD & PCOV & POBS- & MWCD & $\mathrm{PCOV}_{-}$ & POBS- & MWCD- \\
\hline & W & $\mathrm{W}$ & $\mathrm{W}$ & $\mathrm{W}$ & $\mathrm{W}$ & W & W & W & W \\
\hline Group A & 0.1954 & 0.1956 & 0.3937 & 0.0197 & 0.0089 & 0.0923 & 0.2084 & 0.1934 & 0.3826 \\
\hline Group B & 0.3188 & 0.3192 & 0.3227 & 0.0751 & 0.0559 & 0.1246 & 0.3385 & 0.3248 & 0.3354 \\
\hline Group C & 0.3703 & 0.3713 & 0.5351 & 0.0835 & 0.076 & 0.1185 & 0.4073 & 0.3943 & 0.5324 \\
\hline Group D & 0.2805 & 0.2812 & 0.5405 & 0.082 & 0.0782 & 0.1073 & 0.2854 & 0.2793 & 0.5523 \\
\hline Group E & 0.2907 & 0.2908 & 0.3699 & 0.0237 & 0.0232 & 0.1202 & 0.3054 & 0.3094 & 0.3893 \\
\hline
\end{tabular}

based on DetMCD. The estimated values for Cases A, B, C, D, and E are comparable, except for Case A, which is determined to have the best value of 0.0089 for the reweighted version using the POBS approach. From all the contaminated datasets and cases, E is the most accurate because it is close to the uncontaminated dataset in Case A, with a difference of approximately 0.0144 and 0.0143 for the raw and reweighted versions, respectively. By contrast, the MWCD approach performs poorly with the DetMCD algorithm, whereas the other two approaches perform better in both versions. The results of FCH exhibit better performance in Case A, which indicates better performance at the uncontaminated dataset in the raw version, but FastMCD was better at the reweighted version for all the approaches. By contrast, the performances of the PCOV and POBS approaches are approximately close to each other at the FastMCD and FCH estimators for the raw and reweighted versions, but MWCD performs poorly compared with the other approaches.

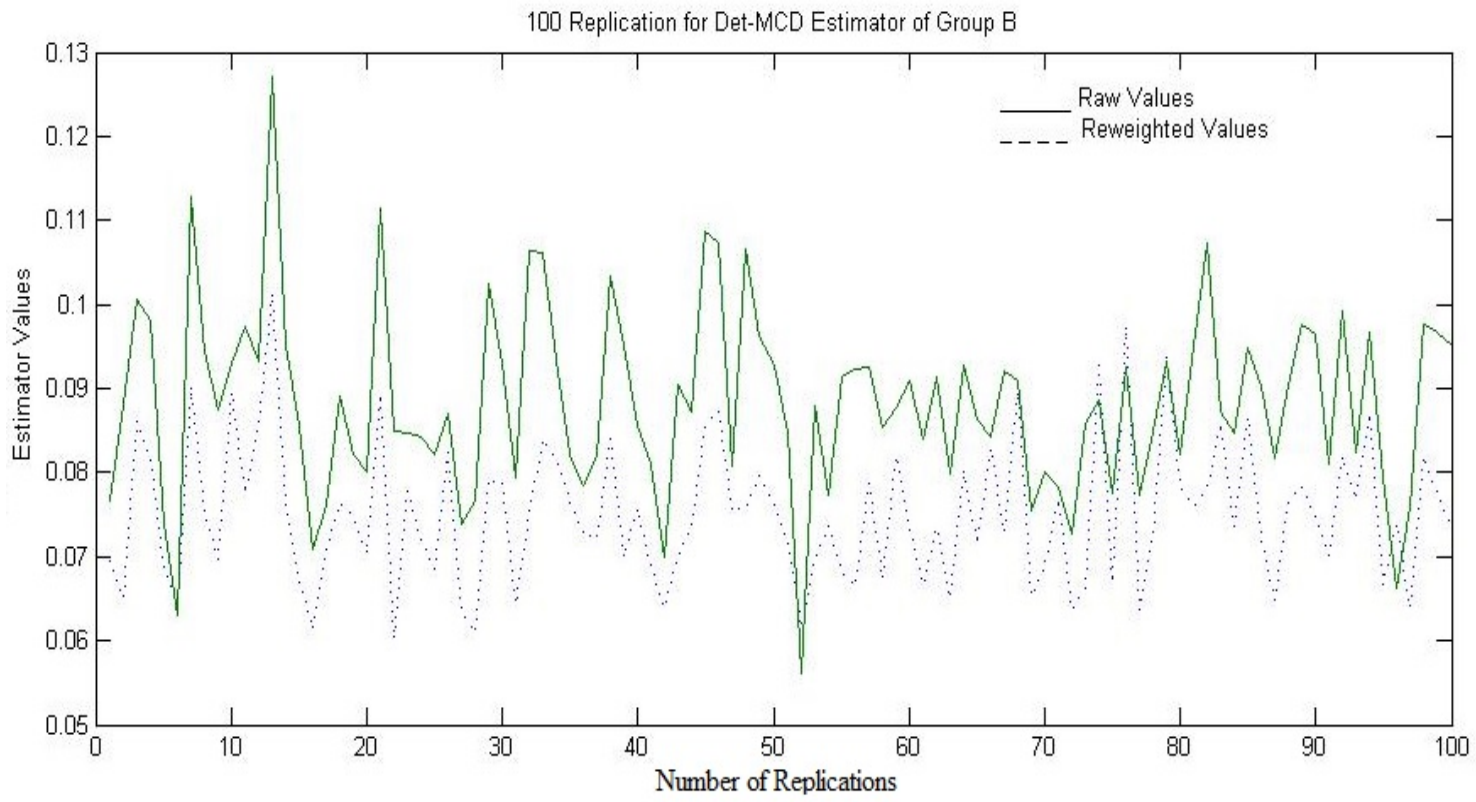

Figure 2: 100 Replications for Det-MCD Estimator of Group B 
Table 3: Misclassification probability estimates for DetMCD, FastMCD, and FCH for the raw version RLDA rules based on actual data (financial ratios for Islamic and conventional banks in Malaysia)

\begin{tabular}{|l|l|l|l|l|l|l|l|l|l|}
\hline Algorithm & FastMCD & \multicolumn{3}{l|}{ DetMCD } & FCH \\
\hline Version & \multicolumn{9}{|l|}{ Raw } \\
\hline Approach & PCOV & POBS & MWCD & PCOV & POBS & MWCD & PCOV & POBS & MWCD \\
\hline MP & 0.214 & 0.1845 & 0.0352 & 0.0182 & 0.06 & 0.2227 & 0.217 & 0.1954 & 0.0932 \\
\hline
\end{tabular}

Table 4: Misclassification probability estimates for DetMCD, FastMCD, and FCH for the reweighted version RLDA rules based on actual data (financial ratios for Islamic and conventional banks in Malaysia)

\begin{tabular}{|l|l|l|l|l|l|l|l|l|l|}
\hline Algorithm & FastMCD & \multicolumn{1}{l|}{ DetMCD } & \multicolumn{1}{l|}{ RFCH } \\
\hline Version & \multicolumn{10}{l|}{ Reweighted } \\
\hline Approach & PCOV- & POBS- & MWCD- & PCOV- & POBS- & MWCD- & PCOV- & POBS- & MWCD- \\
& W & W & W & W & W & W & W & W & W \\
\hline MP & 0.1914 & 0.1745 & 0.0302 & 0.0153 & 0.004 & 0.2246 & 0.1923 & 0.1976 & 0.0763 \\
\hline
\end{tabular}

Figure 2 presents the replications for the first 100 times out of the 500 replications. The figure describes the values of the misclassification probabilities of the discriminate rules based on the DetMCD estimator. The disparity of the misclassification values is shown, particularly for the raw version, where the difference between the minimum and maximum values is considerable compared with that for the reweighted version. In terms of the accuracy of the versions of the DetMCD estimator, the reweighted version is more accurate and efficient than the raw version.

\section{Example of actual data: Islamic and conventional banks in Malaysia}

The financial ratios of Islamic and conventional banks in Malaysia are used as actual data. A total of 271 observations from banks for the period of 2003-2011 are used, where 96 observations are from Islamic banks and 175 observations are from conventional banks. The dataset has 23 financial ratios (variables). The data were collected from the Bankscope database, which converts financial data according to common international standards to facilitate comparisons. RLDA is applied using the three estimators. All the estimators have been used with the three approaches described in the previous section. The results are presented in Table 3 and Table 4 for the raw and reweighted versions, respectively.

Table 3 and Table 4 show the misclassification probabilities for the two types of banks in Malaysia. The DetMCD estimator outperforms the FastMCD and FCH estimators by a significant margin. DetMCD yields more accurate results for the actual data, as expected in the simulation study. However, the reweighted version confirms the high performance in MP estimation. When the three approaches are compared under the two algorithms, the PCOV and POBS approaches perform better with DetMCD than the other estimators and increase the accurate estimation of MP. MWCD performs better with FastMCD and FCH than DetMCD and makes the discriminate rules more efficient and accurate in estimating the misclassification probabilities of Islamic and conventional banks. 


\section{Conclusion}

In this study, we investigated the difference in efficiency among three estimators, namely, DetMCD, FastMCD, and FCH, of location and scatter for RLDA, in which the groups have a common covariance matrix. DetMCD, FCH, and FastMCD are compared based on three approaches to estimate the common scatter matrix. Membership probabilities in a robust structure are estimated by considering only observations of non-outliers. Then, misclassification probabilities for the data are obtained and set into two groups of datasets.

The results of the simulation clearly showed how the robust structure was better and how the DetMCD algorithm for the robust and non-robust structures was better than the FastMCD and FCH algorithms. For the robust structure, DetMCD performed better and was unaffected by outliers. The DetMCD algorithm achieved high efficiency for RLDA and was more accurate than the FastMCD and FCH algorithms. We applied the RLDA rules on actual datasets based on the two algorithms.

The DetMCD algorithm performed well compared with the FastMCD and FCH algorithms; RLDA with DetMCD achieved the highest efficiency. Thus, the DetMCD algorithm increased the accuracy and performance of the LDA model, which indicates more advantages to utilize the model with highly robust estimations. On the basis of the results of the simulation and actual data, the DetMCD algorithm can be used with RLDA in financial research to predict firm failure, bankruptcy, and company distress. It also has applications in other fields, e.g., recognitions.

\section{Acknowledgements}

The authors gratefully acknowledge Qassim University, represented by the Deanship of Scientific on the material support for this research under the number (1475) during the academic year $1437 \mathrm{AH} / 2016 \mathrm{AD}$.

\section{References}

[1] Mufda Jameel Alrawashdeh, Shamsul Rijal Muhammad Sabri, and Mohd Tahir Ismail. Robust linear discriminant analysis with financial ratios in special interval. Applied Mathematical Sciences, 6(121):6021-6034, 2012.

[2] Billor, Nedret, Ali S Hadi, and Paul F Velleman. Bacon: blocked adaptive computationally efficient outlier nominators. Computational Statistics and Data Analysis, $34(3): 279-298,2000$.

[3] Chork, CY, and Peter J Rousseeuw. Integrating a high-breakdown option into discriminant analysis in exploration geochemistry. Journal of Geochemical Exploration, 43(3):191-203, 1992. 
[4] Croux, Christophe, and Catherine Dehon. Analyse canonique base sur des estimateurs robustes de la matrice de covariance. Revue de statistique applique, 50(2):5-26, 2002.

[5] Croux, Christophe, Peter Filzmoser, and Kristel Joossens. Classification efficiencies for robust linear discriminant analysis. Statistica Sinica, 18(2):581-599, 2008.

[6] Croux, Christophe, Sarah Gelper, and Koen Mahieu. Robust exponential smoothing of multivariate time series. Computational Statistics and Data Analysis, 54(12):29993006, 2010.

[7] Croux, Christophe, and Gentiane Haesbroeck. Principal component analysis based on robust estimators of the covariance or correlation matrix: influence functions and efficiencies. Biometrika, 87(3):603-618, 2000.

[8] Devlin, S. J., Gnanadesikan, R., Kettenring, and J. R. Robust estimation of dispersion matrices and principal components. Journal of the American Statistical Association, 76(374):354-362, 1981.

[9] Fekri, M, and Anne Ruiz-Gazen. Robust weighted orthogonal regression in the errorsin-variables model. Journal of the American Statistical Association, 88(1):89-108, 2004.

[10] Hawkins, D.M., Olive, and D.J. Improved feasible solution algorithms for high breakdown estimation. Computational Statistics and Data Analysis, 30:1-11, 1999.

[11] Hawkins, Douglas M, and Geoffrey J McLachlan. High-breakdown linear discriminant analysis. Journal of the American statistical association, 92(437):136-143, 1997.

[12] He, Xuming, and Wing K Fung. High breakdown estimation for multiple populations with applications to discriminant analysis. Journal of Multivariate Analysis, $72(2): 151-162,2000$.

[13] Hubert, Mia, and K Vanden Branden. Robust methods for partial least squares regression. Journal of Chemometrics, 17(10):537-549, 2003.

[14] Hubert, Mia, and Katrien Van Driessen. Fast and robust discriminant analysis. Computational Statistics and Data Analysis, 45(2):301-320, 2004.

[15] Hubert, Mia, and Peter J Rousseeuw. Robust regression with both continuous and binary regressors. Journal of Statistical Planning and Inference, 57(1):153-163, 1997.

[16] Hubert, Mia, Peter J Rousseeuw, and Stefan Van Aelst. High-breakdown robust multivariate methods. Statistical Science, 23(1):92-119, 2008.

[17] Hubert, Mia, Peter J Rousseeuw, and Karlien Vanden Branden. Robpca: a new approach to robust principal component analysis. Technometrics, 47(1):64-79, 2005. 
[18] Hubert, Mia, Peter J Rousseeuw, and Tim Verdonck. A deterministic algorithm for robust location and scatter. Journal of Computational and Graphical Statistics, 21(3):618-637, 2012.

[19] Hubert, Mia, and Sabine Verboven. A robust pcr method for highdimensional regressors. Journal of Chemometrics, 17(89):438-452, 2003.

[20] Maronna, Ricardo A, and Ruben H Zamar. Robust estimates of location and dispersion for high-dimensional datasets. Technometrics, 44(4):307-317, 2002.

[21] David J. Olive and Douglas M. Hawkins. Robust multivariate location and dispersion. Southern Illinois University and University of Minnesota, 2010.

[22] Pison, Greet, and et al. Robust factor analysis. Journal of Multivariate Analysis, 84(1):145-172, 2003.

[23] Rousseeuw and Peter J. Least median of squares regression. Journal of the American statistical association, 79(388):871-880, 1984.

[24] Rousseeuw, Peter J, and Christophe Croux. Alternatives to the median absolute deviation. Journal of the American Statistical Association, 88(424):1273-1283, 1993.

[25] Rousseeuw, Peter J, and Katrien Van Driessen. A fast algorithm for the minimum covariance determinant estimator. Technometrics, 41(3):212-223, 1999.

[26] Rousseeuw, Peter J, and Bert C Van Zomeren. Unmasking multivariate outliers and leverage points. Journal of the American Statistical Association, 85(411):633-639, 1990.

[27] Todorov and Valentin. Robust selection of variables in linear discriminant analysis. Statistical Methods and Applications, 15(3):395-407, 2007.

[28] S. Visuri, H. Oja, and V. Koivunen. Sign and rank covariane matrices. J. Statist. Plann. Inference, 91:557575, 2000.

[29] Wiegand, Patrick, Randy Pell, and Enric Comas. Simultaneous variable selection and outlier detection using a robust genetic algorithm. Chemometrics and Intelligent Laboratory Systems, 98(2):108-114, 2009.

[30] Jianfeng Zhang, David J. Olive, and Ping Ye. Robust covariance matrix estimation with canonical correlation analysis. International Journal of Statistics and Probability, 1(2):119-136, 2012. 